Modeling, Identification and Control, Vol. 29, No. 4, 2008, pp. 131-149

\title{
Straight-Line Target Tracking for Unmanned Surface Vehicles
}

\author{
Morten Breivik ${ }^{1}$ Vegard E. Hovstein ${ }^{2}$ Thor I. Fossen ${ }^{1,3}$
}

\author{
${ }^{1}$ Centre for Ships and Ocean Structures, Norwegian University of Science and Technology, NO-7491 Trondheim, \\ Norway. E-mail: morten.breivik@ieee.org \\ ${ }^{2}$ Maritime Robotics, NO-7010 Trondheim, Norway. E-mail: vegard.hovstein@maritimerobotics.com \\ ${ }^{3}$ Department of Engineering Cybernetics, Norwegian University of Science and Technology, NO-7491 Trondheim, \\ Norway. E-mail: fossen@ieee.org
}

\begin{abstract}
This paper considers the subject of straight-line target tracking for unmanned surface vehicles (USVs). Target-tracking represents motion control scenarios where no information about the target behavior is known in advance, i.e., the path that the target traverses is not defined apriori. Specifically, this work presents the design of a motion control system which enables an underactuated USV to track a target that moves in a straight line at high speed. The motion control system employs a guidance principle originally developed for interceptor missiles, as well as a novel velocity controller inspired by maneuverability and agility concepts found in fighter aircraft literature. The performance of the suggested design is illustrated through full-scale USV experiments in the Trondheimsfjord.
\end{abstract}

Keywords: Target Tracking, Unmanned Surface Vehicles, High Speed Motion, Underactuation, Constant Bearing Guidance, Velocity Control System, Full-Scale Experiments

\section{Introduction}

Since a main pillar of the Norwegian economy is related to oil and gas production, a principal research motivation concerns the commercial offshore market, where unmanned vehicle technology is expected to play a key role in future hydrocarbon exploration and exploitation. Additional applications include surveillance of territorial waters, protection of offshore installations, support of oil and gas activities in Arctic regions, environmental monitoring, and data collection operations aiding marine harvest policies. The use of unmanned vehicles can contribute to reduced personnel costs, improved personnel safety, widened weather window of operations, increased operational precision, and more environmentally friendly activities.

When people hear about such vehicles today, they mostly think about either unmanned aerial vehicles (UAVs), unmanned underwater vehicles (UUVs), or unmanned ground vehicles (UGVs). Little attention has been paid to unmanned surface vehicles (USVs). In fact, only last year did the US Navy release its first USV Master Plan (Navy, 2007), where a USV is defined as a vehicle which displaces water at rest and operates with near continuous contact with the water surface, capable of unmanned operations with varying degrees of autonomy.

However, USVs have actually been developed and operated since World War II, but mostly as drone boats for mine clearance and firing practice. It is only during the last decade that they have been considered for more advanced operations. A majority of the USVs currently under development are found in the US, and the technology is mainly developed for naval purposes. In par- 
ticular, the only industrial-level USVs today are found within the naval segment, mainly for intelligence, surveillance, and reconnaissance (ISR) applications. Most scientific USVs are just experimental platforms, and no applications currently exist in the commercial market. Details about the history, current status, and possible future development of USVs can be found in (Portmann et al., 2002), (Brown, 2004), (Hook, 2006), (Caccia, 2006), (Corfield and Young, 2006), (Bertram, 2008), and (Withington, 2008).

As most other unmanned vehicles, USVs are typically envisioned for use in so-called dirty, dull, and dangerous operations. USV technology harbors a great potential for a number of qualities, including possibilities for new vehicle designs and new concepts of operation. Regarding vehicle designs, current USVs are mostly small, boat-like vehicles that have been adapted from manned vessels originally designed to accommodate human occupants. However, such limitations need not apply to unmanned vehicles (Cooper et al., 2002), which, e.g., can be designed as semi-submersibles for improved stealth and platform stability.

Furthermore, given that USVs are typically small, fast and highly maneuverable vehicles with a large power-to-weight ratio, new motion control concepts must be developed to take advantage of such properties. Traditionally, motion control systems have been developed for fairly large vessels that are not designed for both rapid and precise maneuvering, especially since they spend most of their time in transit through open waters. State-of-the-art solutions for these typically small power-to-weight ratio vessels include course-keeping and course-changing autopilots that provide them with the ability to carry out relatively slow maneuvers (Fossen, 2002). Such autopilots do not suffice for many USV purposes.

Moreover, USVs might cooperate with other unmanned vehicles such as UAVs and UUVs to form large heterogeneous communication and surveillance networks that are able to provide unique situational awareness capabilities. In fact, USVs are unique in the sense that they are able to communicate with vehicles both above and below the sea surface at the same time, capable of acting as relays between underwater vehicles and vehicles operating on land, in the air, or in space. They can also be used to augment the capability of manned surface vessels performing various survey tasks by attaching themselves in purposeful geometric patterns around the manned vessels in order to increase their spatio-temporal survey capacity. Such formation control applications require advanced motion control systems with collision avoidance (CA) functionality. In turn, CA functionality requires both sense and avoid abilities, i.e., access to both global and local informa-

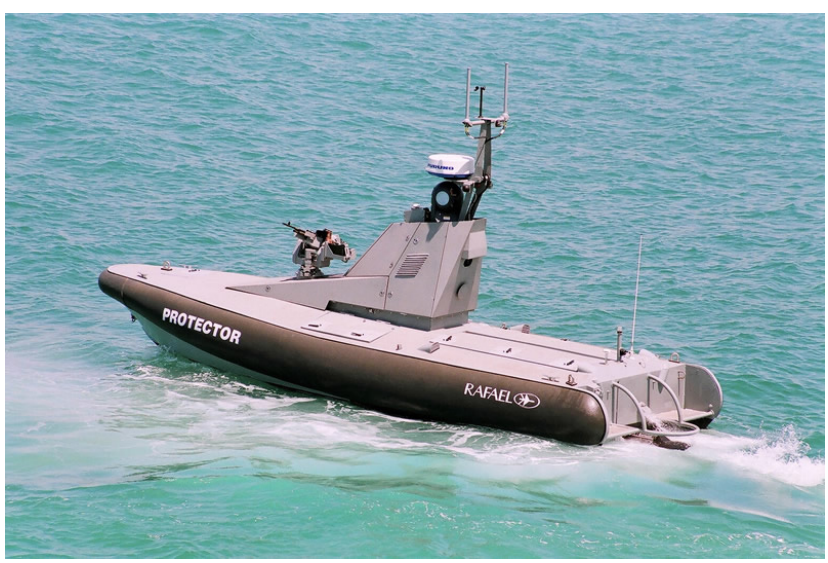

Figure 1: One example of an industrial-level USV is the remotely controlled Protector, developed by Rafael of Israel. This vehicle is a 9 $m$ long rigid-hulled inflatable boat equipped with water jet propulsion that enables operations of up to $20 \mathrm{~m} / \mathrm{s}$ (i.e., approximately 40 knots).

tion about the environment, as well as superior maneuverability through powerful actuators. Collision avoidance systems for USVs are reported by Benjamin et al. (2006), Larson et al. (2007), and Loe (2008).

In any case, the future prosperity of USV applications depends on the development of a legal framework that renders possible unmanned operations in traditionally manned areas. Operational possibilities within the current framework of the International Maritime Organization (IMO) are reported in (Gibbons and Wilson, 2008), while future inspiration can be sought from ongoing work that is performed in the US regarding UAVs (DeGarmo and Nelson, 2006).

The main contribution of this paper is the development of a motion control system which facilitates highspeed target tracking for underactuated USVs. Specifically, the suggested motion control system consists of two main subsystems, i.e., a guidance system and a velocity control system. The guidance system employs a missile technique known as constant bearing guidance to calculate a desired velocity which enables the USV to track a moving target, while the velocity control system consists of speed and steering controllers that make the actual USV velocity adhere to the desired velocity commanded by the guidance system. The result is a simple yet advanced motion control system which requires a minimum of system identification and tuning tests to be carried out. Full-scale experiments involving an underactuated USV and a target moving in a straight line at high speed are used to illustrate the performance of the proposed motion control scheme. 


\section{Motion Control Fundamentals}

This section introduces some fundamental motion control concepts, including operating spaces, vehicle actuation properties, motion control scenarios, as well as the motion control hierarchy. The material is adapted from (Breivik and Fossen, 2008).

\subsection{Operating Spaces}

To enable purposeful definitions of motion control scenarios it is necessary to distinguish between different operating spaces. In this regard, the two most fundamental operating spaces are the work space and the configuration space. The work space is also known as the operational space (Sciavicco and Siciliano, 2002), and represents the physical space in which a vehicle moves. On the other hand, the configuration space, also known as the joint space (Sciavicco and Siciliano, 2002), is constituted by the set of variables sufficient to specify all points of a rigid-body vehicle in the work space (LaValle, 2006). Each configuration variable is called a degree of freedom (DOF).

\subsection{Vehicle Actuation Properties}

The type, amount, and distribution of vehicle thrust devices and control surfaces, hereafter commonly referred to as actuators, determine the actuation properties of a vehicle. We mainly distinguish between two qualitatively different actuation properties, namely full actuation and underactuation. A fully actuated vehicle is able to independently control the motion of all its DOFs simultaneously, while an underactuated vehicle is not. Thus, an underactuated vehicle is generally unable to achieve arbitrary tasks in its configuration space. However, it will be able to achieve tasks in the work space as long as it can freely project its main thrust in this space, e.g., through a combination of thrust and attitude control. In fact, this principle is the mode by which most vehicles that move through a fluid operate, from missiles to ships. Even if these vehicles had the ability to roam the work space with an arbitrary attitude, they would usually expend an unnecessary amount of energy by doing so. In practice, most vehicles are underactuated in their configuration space at high speeds, and are forced to maneuver in an energy-efficient manner. Ships are typically underactuated above $1.5-2 \mathrm{~m} / \mathrm{s}(3-4$ knots $)$ since the actuators that facilitate full actuation are ineffective above such speeds (Kongsberg Maritime, 2006).

\subsection{Motion Control Scenarios}

In the traditional control literature, motion control scenarios are typically divided into the following categories: point stabilization, trajectory tracking, and path following. More recently, the concept of maneuvering has been added to the fold as a means to bridge the gap between trajectory tracking and path following (Skjetne et al., 2004a). These scenarios are often defined by motion control objectives that are given as configuration-space tasks, which are best suited for fully actuated vehicles. Also, the scenarios typically involve desired motion that has been defined apriori in some sense. Little seems to be reported about tracking of target points for which only instantaneous motion information is available. However, in (Breivik and Fossen, 2008), both apriori and non-apriori scenarios are considered, and all the motion control objectives are given as work-space tasks. Thus, the scenarios cover more broadly, and are also suited for underactuated vehicles. Specifically, these scenarios encompass:

- Target tracking: The control objective is to track the motion of a target that is either stationary (similar to point stabilization) or that moves such that only its instantaneous motion is known, i.e., such that no information about the future target motion is available. Thus, in this case it is impossible to separate the spatio-temporal constraint associated with the target into two separate constraints.

- Path following: The control objective is to follow a predefined path, which only involves a spatial constraint. No restrictions are placed on the temporal propagation along the path.

- Path tracking: The control objective is to track a target that moves along a predefined path (similar to trajectory tracking). Consequently, it is possible to separate the target-related spatio-temporal constraint into two separate constraints. Still, this scenario can be viewed as a target-tracking scenario and handled with target-tracking methods, thus disregarding any apriori path information that is available.

- Path maneuvering: The control objective is to employ knowledge about vehicle maneuverability constraints to feasibly negotiate (or optimize the negotiation of) a predefined path. Path maneuvering thus represents a subset of path following, but is less constrained than path tracking since spatial constraints always take precedence over temporal constraints. Path-maneuvering methods can also be used to handle path-tracking scenarios. 


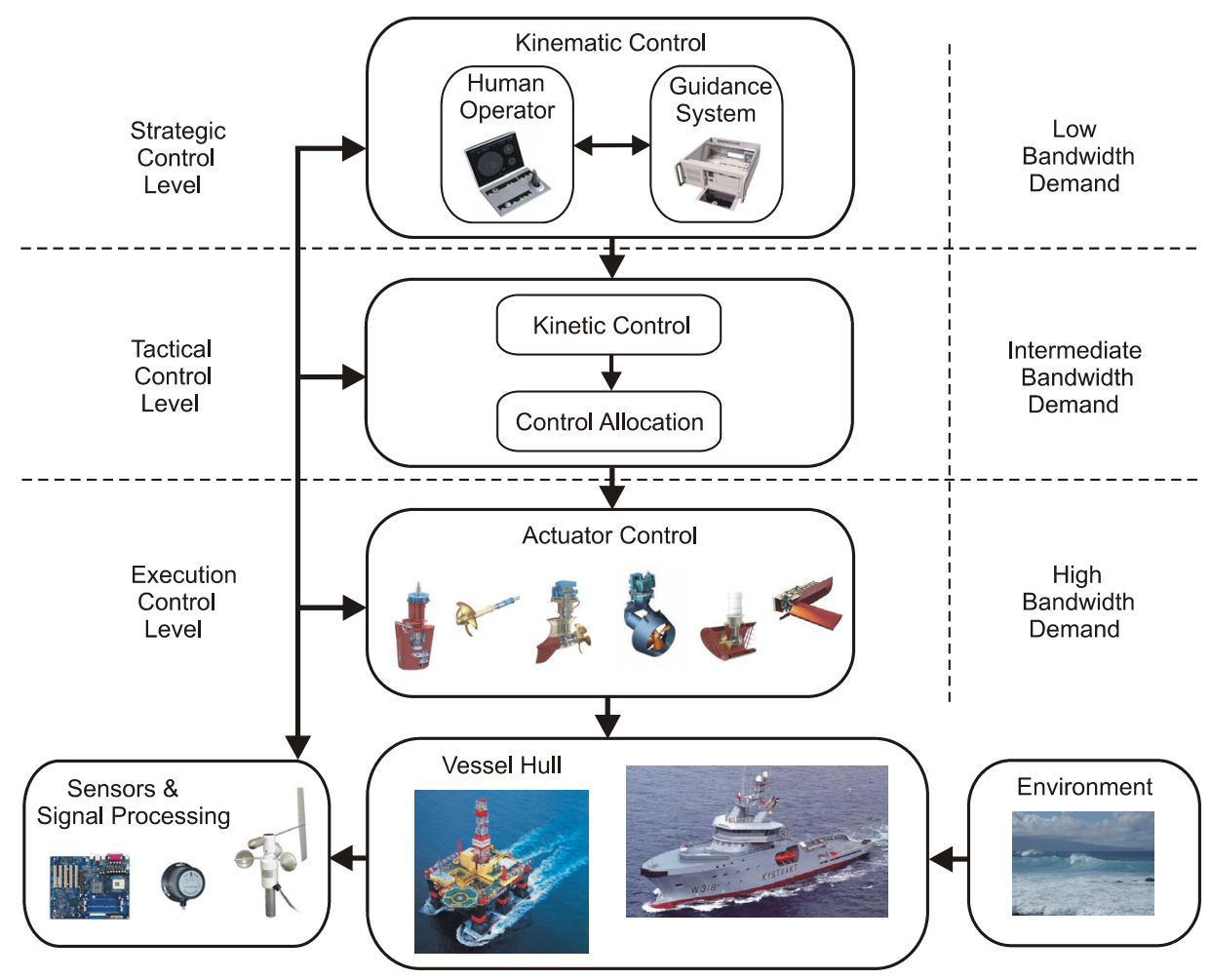

Figure 2: The motion control hierarchy of a marine surface vessel nominally consists of strategic, tactical, and execution levels of control. Additional levels are required to achieve full autonomy.

The work in this paper is concerned with the targettracking scenario. Specifically, the motion control objective is to track a target which moves at high speed, and for which no future motion information is available. The only accessible target information is its instantaneous position and velocity.

\subsection{Motion Control Hierarchy}

The purpose of a motion control system is to enable a vehicle to fulfill its assigned motion control objective, and can be conceptualized to involve at least three control levels in a hierarchical structure. Figure 2 illustrates the typical components of a marine motion control system, encompassing strategic, tactical, and execution levels of control (Valavanis et al., 1997). All the involved building blocks represent autonomy-enabling technology, but more instrumentation and additional control levels are required to attain full autonomy.

At the top, we find the strategic control level. Also termed the kinematic control level, it is responsible for prescribing vehicle velocity commands needed to achieve motion control objectives in the work space. Thus, in this paper, kinematic control is equivalent to work-space control, and kinematic controllers are referred to as guidance laws. This level purely considers the geometric aspects of motion, without reference to the forces and moments that generate such motion.

Next, the tactical level encompass kinetic controllers, which do consider how forces and moments generate vehicle motion. These controllers are typically designed by model-based methods, and must handle both parametric model uncertainties and environmental disturbances. For underactuated vehicles, they must actively employ the vehicle attitude as a means to achieve the velocities prescribed by the guidance module. The intermediate control level also contains a control allocation block which distributes the kinetic control commands among the various vehicle actuators.

At the bottom, the individual actuator controllers constitute the execution level, ensuring that the actuators behave as requested by the intermediate control module, and ultimately that the vehicle moves as prescribed by the guidance laws.

This paper focuses on the strategic and tactical control levels, and proposes corresponding guidance and velocity control systems that enable an underactuated USV to fulfill a target-tracking motion control objective. Note that having well-functioning tactical- and execution-level controllers, strategic-level controllers can be exchanged in a modular manner to achieve different motion control objectives. 


\section{Motion Control System Design}

Only a handful of papers currently deal with motion control system design for USVs. These include (Ebken et al., 2005), where motion control technology originally developed for UGVs is used to rapidly achieve basic motion control functionality for USVs, including modes for remote control and waypoint navigation; (Majohr and Buch, 2006), which details the development of a small USV intended to carry out high-precision survey operations in shallow waters, employing a steering controller based on traditional autopilot design methods; (Doucy and Ghozlan, 2008), where qualitative descriptions of advanced motion control capabilities for USVs are given, including dynamic positioning, wave management, obstacle avoidance, and fleet control; (Caccia et al., 2008a), which shows how conventional motion control techniques can be applied to make a small USV equipped with only a GPS antenna and a compass perform auto-heading, auto-speed, and straight-line path-following tasks; and (Naeem et al., 2008), where an LQG-based autopilot is proposed for a USV intended for environmental monitoring and pollutant tracking. Common features of these works are that they employ traditional control techniques and mostly consider low-speed operations in calm water.

So far, no results seem to have been reported on highspeed target-tracking for underactuated vessels. Precision control for target-tracking scenarios is currently achieved by dynamic positioning systems that require fully actuated vessels and thus concerns low speeds (Sørensen et al., 2001). As an attempt to improve this situation, the development of a novel motion control system for an underactuated USV whose assignment is to track a high-speed target is detailed in the following. The proposed design combines a well-known guidance technique with a novel velocity control system consisting of surge speed and yaw rate controllers. Literature on missile guidance, fighter aircraft, and marine vehicles has inspired the approach, which inherently takes saturation limits in the actuator system into account. The suggested design is illustrated for a small planing monohull made from aluminum designated the Kaasbøll USV, which was the first test platform of the Trondheim-based company Maritime Robotics.

\subsection{Guidance System}

Guidance represents a fundamental methodology which transcends specific vehicle applications (Draper, 1971), and is concerned with the transient motion behavior related to the achievement of motion control objectives (Shneydor, 1998). For this reason, guidance laws are typically stated at a kinematic level, only considering the fundamental geometric aspects of the scenarios of interest. In what follows, three missile guidance techniques applicable to target-tracking scenarios are presented, and the material is adapted from (Breivik and Fossen, 2008).

Representing a kinematic vehicle by its planar position $\mathbf{p}(t) \triangleq[x(t), y(t)]^{\top} \in \mathbb{R}^{2}$ and velocity $\mathbf{v}(t) \triangleq$ $\mathrm{d} \mathbf{p}(t) / \mathrm{d} t \triangleq \dot{\mathbf{p}}(t) \in \mathbb{R}^{2}$, stated relative to some stationary reference frame, and denoting the position of the target by $\mathbf{p}_{\mathrm{t}}(t) \triangleq\left[x_{\mathrm{t}}(t), y_{\mathrm{t}}(t)\right]^{\top} \in \mathbb{R}^{2}$, the control objective of a target-tracking scenario can be stated as

$$
\lim _{t \rightarrow \infty}\left(\mathbf{p}_{\mathrm{t}}(t)-\mathbf{p}(t)\right)=\mathbf{0}
$$

where $\mathbf{p}_{\mathrm{t}}(t)$ is either stationary or moving by a (nonzero and bounded) velocity $\mathbf{v}_{\mathrm{t}}(t) \triangleq \dot{\mathbf{p}}_{\mathrm{t}}(t) \in \mathbb{R}^{2}$.

Also, let the speed of the kinematic vehicle be denoted $U(t) \triangleq|\mathbf{v}(t)| \triangleq \sqrt{\dot{x}(t)^{2}+\dot{y}(t)^{2}} \geq 0$, while the course angle is denoted $\chi(t) \triangleq \operatorname{atan} 2(\dot{y}(t), \dot{x}(t)) \in \mathbb{S} \triangleq$ $[-\pi, \pi]$, where atan $2(y, x)$ is the four-quadrant version of $\arctan (y / x) \in\langle-\pi / 2, \pi / 2\rangle$. Correspondingly, the speed and course of the target are denoted $U_{\mathrm{t}}(t)$ and $\chi_{\mathrm{t}}(t)$, where $U_{\mathrm{t}}(t) \in\langle 0, \infty\rangle$.

Concerning tracking of moving targets, the missile guidance community commonly refers to the object that is supposed to destroy another object as either a missile, an interceptor, or a pursuer. Conversely, the threatened object is typically called a target or an evader. In the following, the neutral designations interceptor and target will be used when presenting 3 fundamental guidance strategies, namely line of sight, pure pursuit, and constant bearing. These guidance strategies are referred to as the classical guidance laws, and the associated geometric principles are illustrated in Figure 3.

\subsubsection{Line of Sight Guidance}

Line of sight (LOS) guidance is classified as a threepoint guidance scheme since it involves a (typically stationary) reference point in addition to the interceptor and the target. The LOS denotation stems from the fact that the interceptor is supposed to achieve an intercept by constraining its motion along the line of sight between the reference point and the target. LOS guidance has typically been employed for surface-to-air missiles, often mechanized by a ground station which illuminates the target with a beam that the guided missile is supposed to ride, also known as beam-rider guidance. The LOS guidance principle is illustrated in Figure 3, where the associated velocity command is represented by a vector pointing to the left of the target. 


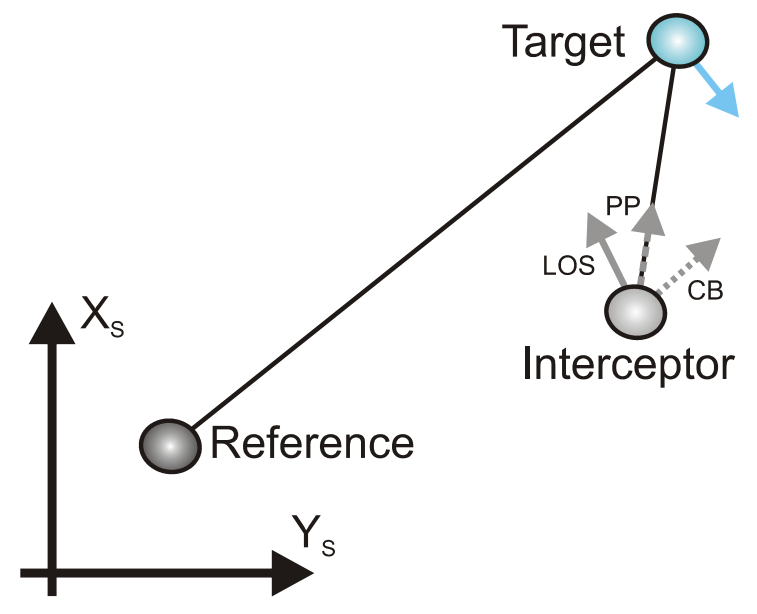

Figure 3: The interceptor velocity commands associated with the classical guidance principles line of sight (LOS), pure pursuit (PP), and constant bearing $(\mathrm{CB})$.

\subsubsection{Pure Pursuit Guidance}

Pure pursuit (PP) guidance belongs to the two-point guidance schemes, where only the interceptor and the target are considered in the engagement geometry. Simply put, the interceptor is supposed to align its velocity along the line of sight between the interceptor and the target. This strategy is equivalent to a predator chasing a prey in the animal world, and very often results in a tail chase. PP guidance has typically been employed for air-to-surface missiles. The PP guidance principle is represented in Figure 3 by a vector pointing directly at the target.

\subsubsection{Constant Bearing Guidance}

Constant bearing $(\mathrm{CB})$ guidance is also a two-point guidance scheme, with the same engagement geometry as PP guidance. However, in a CB engagement the interceptor is supposed to align the relative interceptortarget velocity along the line of sight between the interceptor and the target. This goal is equivalent to reducing the LOS rotation rate to zero such that the interceptor perceives the target at a constant bearing, closing in on a direct collision course. CB guidance is often referred to as parallel navigation, and has typically been employed for air-to-air missiles. Also, the $\mathrm{CB}$ rule has been used for centuries by mariners to avoid collisions at sea; steering away from a situation where another vessel approaches at a constant bearing. Thus, guidance principles can just as well be applied to avoid collisions as to achieve them. The CB guidance principle is indicated in Figure 3 by a vector pointing to the right of the target.

The most common method of implementing CB guidance is to make the rotation rate of the interceptor velocity directly proportional to the rotation rate of the interceptor-target LOS, which is widely known as proportional navigation (PN). However, CB guidance can also be implemented through the direct velocity assignment

$$
\mathbf{v}(t)=\mathbf{v}_{\mathrm{t}}(t)+\mathbf{v}_{\mathrm{a}}(t),
$$

where $\mathbf{v}_{\mathrm{a}}(t)$ is the velocity with which the interceptor approaches the target, for example chosen as

$$
\mathbf{v}_{\mathrm{a}}(t)=\kappa(t) \frac{\tilde{\mathbf{p}}(t)}{|\tilde{\mathbf{p}}(t)|}
$$

since CB guidance is considered. Here,

$$
\tilde{\mathbf{p}}(t) \triangleq \mathbf{p}_{\mathbf{t}}(t)-\mathbf{p}(t)
$$

is the interceptor-target line-of-sight vector, $|\tilde{\mathbf{p}}(t)|=$ $\sqrt{\tilde{\mathbf{p}}(t)^{\top} \tilde{\mathbf{p}}(t)} \geq 0$ is the Euclidean length of this vector, and $\kappa(t)>0$ can be chosen as

$$
\kappa(t)=U_{\mathrm{a}, \max }(t) \frac{|\tilde{\mathbf{p}}(t)|}{\sqrt{\tilde{\mathbf{p}}(t)^{\top} \tilde{\mathbf{p}}(t)+\triangle_{\tilde{\mathbf{p}}}^{2}}},
$$

where $U_{\mathrm{a}, \max }(t)>0$ specifies the maximum approach speed toward the target, and $\triangle_{\tilde{p}}>0$ influences the transient interceptor-target behavior. This particular implementation of $\mathrm{CB}$ guidance seems to first have been suggested in (Breivik et al., 2006), and later also used in (Breivik and Fossen, 2007).

The direct velocity assignment (2) means that in addition to assigning the target speed, which nullifies the relative velocity flow between the interceptor and the target, a relative approach velocity is assigned along the interceptor-target line-of-sight vector to ensure a smooth rendezvous, bounded by the maximum approach speed of $U_{\mathrm{a}, \max }(t)$ for large $|\tilde{\mathbf{p}}(t)|$ relative to $\triangle_{\tilde{\mathrm{p}}}$. The concept is illustrated in Figure 4, where it can also be seen that $\mathrm{CB}$ guidance becomes equal to PP guidance for a stationary target, i.e., the basic difference between the two guidance schemes is whether the target velocity is used as a kinematic feedforward or not. This difference is vital for underactuated vehicles, which cannot change the direction of their velocity faster than they can turn.

For our application, we only consider moving targets, i.e., targets with positive speed $U_{\mathrm{t}}(t) \geq U_{\mathrm{t}, \text { min }}>0$. Thus, we choose to employ constant bearing guidance, implemented through (2) with (3) and (5), such that

$$
\mathbf{v}(t)=\mathbf{v}_{\mathbf{t}}(t)+U_{\mathrm{a}, \max }(t) \frac{\tilde{\mathbf{p}}(t)}{\sqrt{\tilde{\mathbf{p}}(t)^{\top} \tilde{\mathbf{p}}(t)+\triangle_{\tilde{\mathbf{p}}}^{2}}},
$$




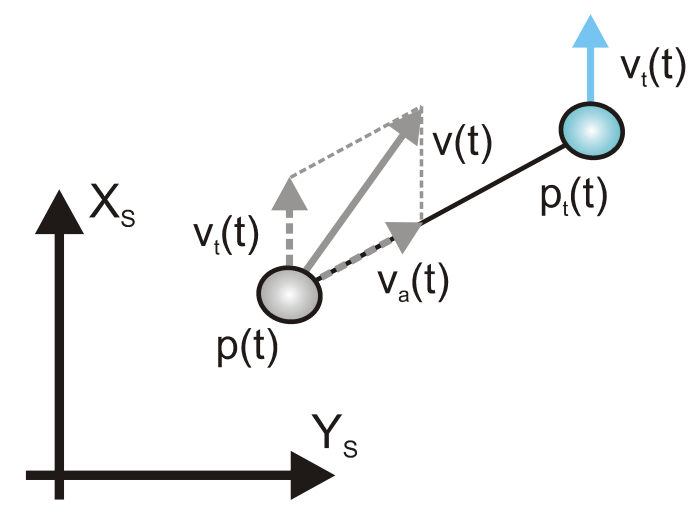

Figure 4: The direct velocity assignment associated with CB guidance.

which means that the target will be pursued at a maximum speed of $U_{\mathrm{t}}(t)+U_{\mathrm{a}, \max }(t)$, which ramps down to $U_{\mathrm{t}}(t)$ when the interceptor-target distance decreases below $\triangle_{\tilde{p}}$ toward zero and rendezvous. In this regard, the choice of $\triangle_{\tilde{p}}$ becomes essential since this parameter explicitly shapes the speed transition between pursuit and rendezvous. For example, too small a value might give too sharp a transition. Figure 5 illustrates the associated speed assignment as a function of interceptortarget distance for movement purely along the x-axis.

This guidance strategy is well suited for underactuated vehicles since an overshoot in position merely reduces the commanded speed below $U_{\mathrm{t}}(t)$ instead of commanding an instantaneous 180 degree turn in the velocity, as would be the case for the pure pursuit strategy. Since the minimum commanded speed is equal to $U_{\mathrm{t}}(t)-U_{\mathrm{a}, \max }(t)$, we must choose $U_{\mathrm{a}, \max }(t)<U_{\mathrm{t}}(t)$ to ensure steerability through forward motion at all times. Specifically, a suitable choice could be

$$
U_{\mathrm{a}, \max }(t)=\zeta U_{\mathrm{t}}(t), \zeta<1
$$

assuming that the interceptor has a speed advantage over the target, i.e., such that $U_{\max }>U_{\mathrm{t}}(t)+$ $U_{\mathrm{a}, \max }(t)=(1+\zeta) U_{\mathrm{t}}(t)$ at all times.

Finally, note that for a real vehicle, the velocity (6) cannot be assigned directly and achieved instantaneously, but rather represents a desired velocity $\mathbf{v}_{\mathrm{d}}(t)$ which the vehicle must attain through the use of a velocity control system, whose design is the topic of the next section.

\subsection{Velocity Control System}

This section details the development of a velocity control system that enables an underactuated USV to achieve the velocity command (6) required to attain the target-tracking motion control objective (1). Hence,

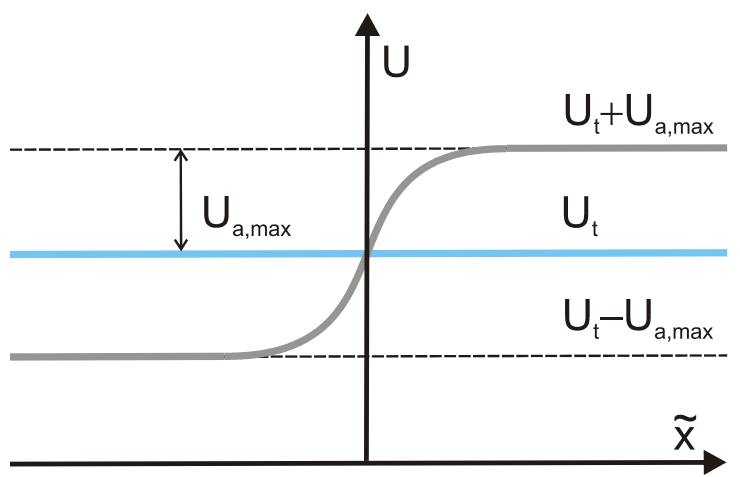

Figure 5: Interceptor speed assignment for movement along the x-axis.

denoting the velocity error as

$$
\tilde{\mathbf{v}}(t) \triangleq \mathbf{v}_{\mathrm{d}}(t)-\mathbf{v}(t)
$$

where $\mathbf{v}_{\mathrm{d}}(t)$ is equal to $(6)$ and $\mathbf{v}(t)$ is the actual USV velocity, the velocity control objective becomes

$$
\lim _{t \rightarrow \infty} \tilde{\mathbf{v}}(t)=\mathbf{0}
$$

In particular, since we consider underactuated USVs, the velocity controller is decomposed into a surge speed controller and a yaw rate controller in a polar coordinate fashion. The design is illustrated for a vehicle named the Kaasbøll USV, and the principal goal is to develop a simple yet advanced velocity control system which requires a minimum of system identification and tuning tests to be carried out.

\subsubsection{The Kaasbøll USV}

The USV that was used as a test platform for the experiments reported in this paper is a modified Kaasbøll 19 boat, which is a $5.75 \mathrm{~m}$ (19 feet) planing monohull made of aluminum produced by Kaasbøll Boats from Hitra near Trondheim, Norway. A width of 2.12 $m$ ensures sufficient space for two people manning the center console of the boat during sea trials. The USV is equipped with an off-the-shelf Evinrude $50 \mathrm{E}$-Tec outboard engine providing $50 \mathrm{hp}$, which gives it a top speed of about $10 \mathrm{~m} / \mathrm{s}$ (approximately 20 knots) in calm water with two people aboard. This propulsion solution corresponds to a propeller and rudder actuator setup, which means that the USV is unactuated in sway. The navigation system relies on a Seapath 20 NAV solution made by Kongsberg Seatex, which replaces several vessel instruments with a single navigation package that outputs position, heading, velocity, and rate of turn (Kongsberg Seatex, 2006). The USV is also equipped with an onboard computer (OBC) 


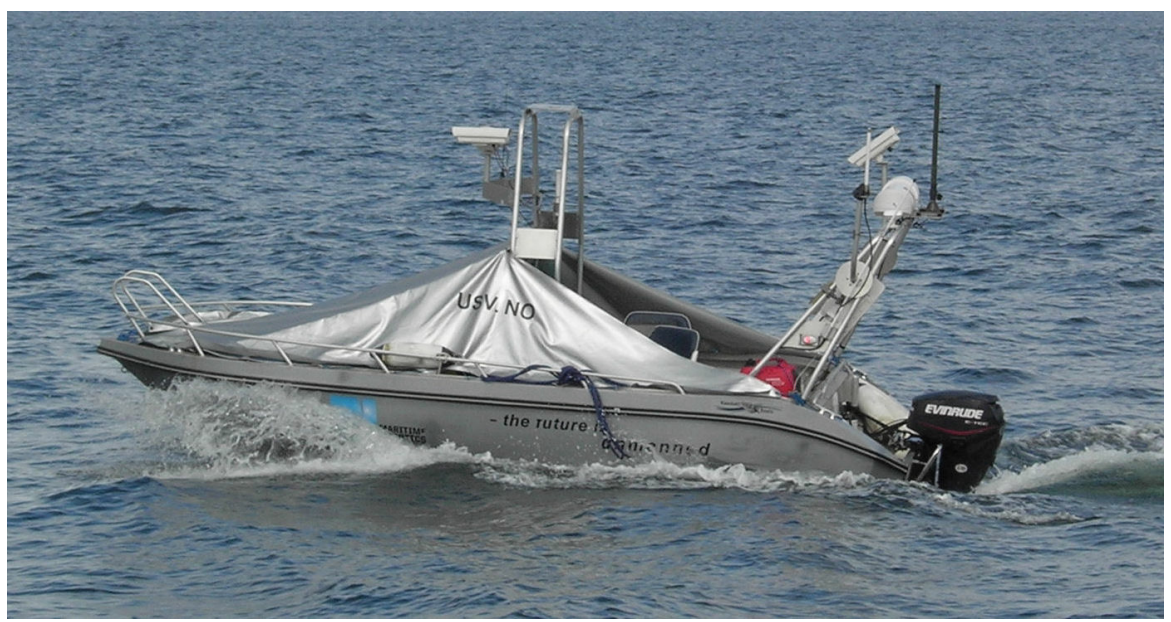

Figure 6: The Kaasbøll USV operating in the Trondheimsfjord.

that provides a rapid prototyping environment with Matlab/Simulink-compliant software. Execution-level proportional controllers ensure that the E-Tec engine responds effectively to throttle commands in the region $\tau_{\mathrm{c}} \in[-100 \%, 100 \%]$ and rudder commands in the region $\delta_{\mathrm{c}} \in[-0.2618 \mathrm{rad}, 0.2618 \mathrm{rad}]$. The fully equipped USV is shown in Figure 6. Due to safety considerations and requirements from the port authorities in Trondheim, all motion control experiments in the Trondheimsfjord are performed with at least two persons aboard the USV.

\subsubsection{Modeling Considerations}

The availability of mathematical models of marine vessels are essential for both control design and simulation study purposes. A standard 3 DOF dynamic model, representing the horizontal surge, sway, and yaw modes, can be found in (Fossen, 2002), and consists of the kinematics

$$
\dot{\boldsymbol{\eta}}=\mathbf{R}(\psi) \boldsymbol{\nu}
$$

and the kinetics

$$
\mathbf{M} \dot{\boldsymbol{\nu}}+\mathbf{C}(\boldsymbol{\nu}) \boldsymbol{\nu}+\mathbf{D}(\boldsymbol{\nu}) \boldsymbol{\nu}=\boldsymbol{\tau}+\mathbf{R}(\psi)^{\top} \mathbf{b},
$$

where $\boldsymbol{\eta} \triangleq[x, y, \psi]^{\top} \in \mathbb{R}^{2} \times \mathbb{S}$ represents the earth-fixed pose (i.e., position and heading); $\boldsymbol{\nu} \triangleq[u, v, r]^{\top} \in \mathbb{R}^{3}$ represents the vessel-fixed velocity; $\mathbf{R}(\psi) \in S O(3)$ is the transformation matrix

$$
\mathbf{R}(\psi) \triangleq\left[\begin{array}{ccc}
\cos \psi & -\sin \psi & 0 \\
\sin \psi & \cos \psi & 0 \\
0 & 0 & 1
\end{array}\right]
$$

that transforms from the vessel-fixed frame to the earth-fixed frame; $\mathbf{M}$ is the inertia matrix; $\mathbf{C}(\boldsymbol{\nu})$ is the centrifugal and coriolis matrix; while $\mathbf{D}(\boldsymbol{\nu})$ is the hydrodynamic damping matrix. The system matrices satisfy the properties $\mathbf{M}=\mathbf{M}^{\top}>0, \mathbf{C}=-\mathbf{C}^{\top}$ and $\mathbf{D}>0$. The vessel-fixed propulsion forces and moment is represented by $\boldsymbol{\tau}$, while $\mathbf{b}$ represents low-frequency, earth-fixed environmental disturbances. Details concerning this model can also be found in (Skjetne et al., 2004b) and (Fossen, 2005).

Most papers considering nonlinear motion control for underactuated marine surface vessels typically use some variant of the model (10)-(11), and assume that the model parameters are either perfectly known or known with only a small degree of uncertainty, see, e.g., (Breivik and Fossen, 2004), (Børhaug and Pettersen, 2005), (Do and Pan, 2006), (Fredriksen and Pettersen, 2006), and (Aguiar and Hespanha, 2007). In practice, it can be quite hard to obtain the parameter values required to populate (11), especially with regard to the hydrodynamic damping matrix. Furthermore, the model is only valid for displacement vessels that operate in a certain part of the speed regime, and does not hold for semi-displacement or planing vessels operating at a large Froude number. This number is a dimensionless parameter defined as

$$
F n \triangleq \frac{U}{\sqrt{L g}},
$$

where $U$ is the vessel speed, $L$ is the submerged vessel length, and $g$ is the acceleration of gravity (Faltinsen, 2005). According to Fossen (2005), the stated 3 DOF model is only valid for $F n \leq 0.3$, which corresponds to a speed of only $2.25 \mathrm{~m} / \mathrm{s}$ for the USV under consideration. However, such a vehicle can operate at much larger Froude numbers, even into the planing region, which is defined for $F n \geq 1.0-1.2$.

A notable exception to the conventional model-based 


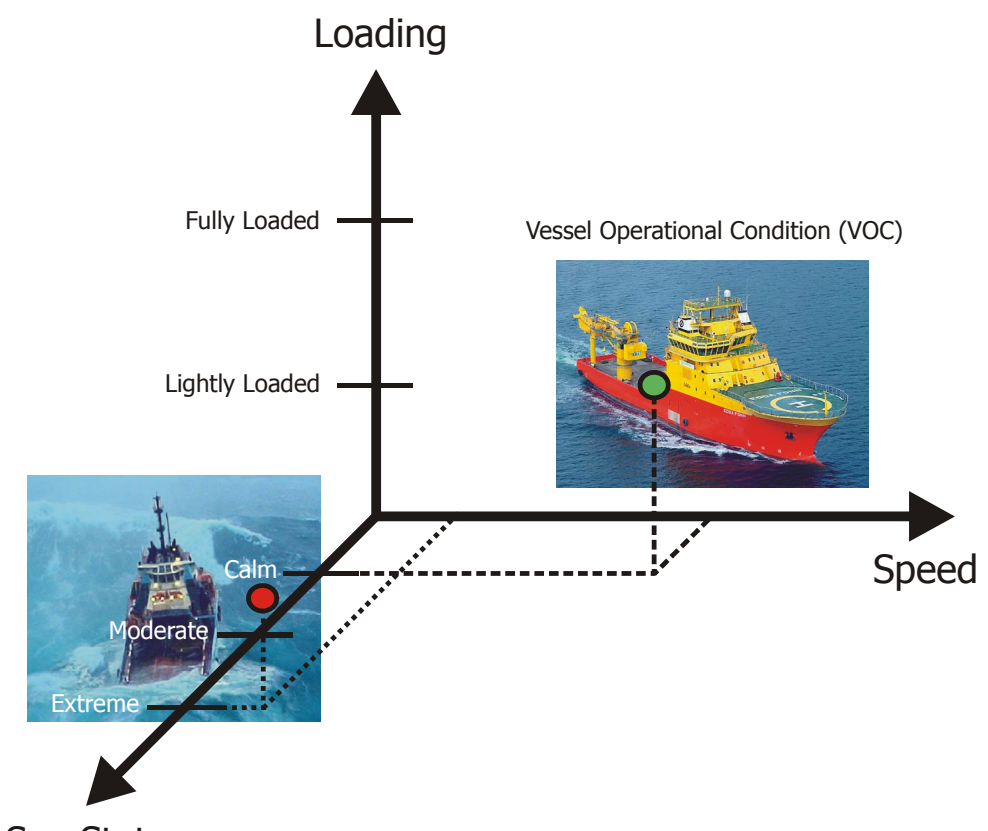

Sea State

Figure 7: The main axes of speed, sea state, and loading constituting the vessel operational condition (VOC) space. Adapted from (Perez et al., 2006).

approach is reported in (Caccia et al., 2008b), where a more control-oriented scheme is suggested. Likewise, we do not use the kinetic model (11) in our design, but employ a more straightforward approach inspired by concepts from literature on fighter aircraft. This scheme is detailed in the following.

\subsubsection{Maneuverability and Agility}

The terms maneuverability and agility are defined in several different ways in literature on fighter aircraft (Paranjape and Ananthkrishnan, 2006), where they are essential for describing close-combat fighting abilities. Similarly, we want to employ such concepts when developing velocity control systems for high-powered USVs. In particular, we choose to subscribe to the definitions used by Beck and Cord (1995), where maneuver performance is defined as a measure of steady maneuver capability and agility is defined as a measure of the ability to transition between steady maneuvers. Consequently, the relevant maneuver states of a surface vehicle include the surge speed $u$, the sway speed $v$, and the yaw rate $r$. These variables determine how fast the vessel can move on the sea surface, i.e., traverse the pose space. The agility of a vessel then describes how fast it can transition between its maneuver states. Various tests can be carried out to determine the maneuverability and agility of a vehicle, and in the following such tests and their results are reported for the Kaasbøll USV.
Maneuverability Tests Several factors determine the maneuverability of a vehicle, but the most important one for control purposes is the relationship between the actuator inputs and the maneuver states. All actuators are ultimately controlled by either a voltage or a current signal, such that their capacity can be conveniently represented in the range $[-100 \%, 100 \%]$ (when abstracting away the actual signal range), where $100 \%$ represents maximum input. For a vehicle whose actuator setup corresponds to that of having a sternmounted propeller and rudder, tests can be carried out in which the control signal for both actuators are applied in steps to cover their entire signal range while simultaneously recording the steady response of the maneuver states. Then, by using, e.g., least-squares curve fitting to the obtained data sets, analytic relationships between the control inputs and the maneuver states can be achieved. The result will ultimately constitute a 5-dimensional surface - a maneuver map - in the combined input (propeller, rudder) and output (surge, sway, and yaw speeds) space, which is the input-output surface that the vessel nominally will be able to traverse.

Furthermore, the tests should be carried out in ideal conditions, i.e., for minimal environmental disturbances (such as wind, waves, and currents) and for nominal loading conditions. The results can then be used to design a feedforward controller that will be able to achieve any allowable set of speeds by simply 
allocating the required control inputs derived from the maneuver map. Feedback terms must also be added to take care of any discrepancies between the nominal maneuver map and the actual situation, resulting, e.g., from changing environmental conditions or off-nominal loading conditions. Hence, the feedforward terms handle operations in the nominal part of the vessel operational condition (VOC) space, while the feedback terms enlarge this operational area by adding robustness against modeling errors, parametric uncertainties, and disturbances. See Figure 7 for an illustration of the main axes in the VOC space.

Since we only consider straight-line target-tracking scenarios, it is not necessary to derive a maneuver map which includes the rudder input and the sway and yaw outputs. Hence, we only consider the relationship between the throttle input and the surge output. Accordingly, the USV maneuverability tests consisted of applying throttle inputs from $0 \%$ to $100 \%$ in steps of $10 \%$ for zero rudder, and recording the corresponding steady-state surge speeds. Negative throttle was not considered relevant.

The tests showed that a throttle input of less than $40 \%$ was barely recognizable on the surge speed output, which means that the range $0-40 \%$ in practice constitutes a dead band. Also, for throttle above $80 \%$, the USV transitioned from the displacement region into the semi-displacement and planing regions, where it is much harder to achieve precision control of the speed. Consequently, we only consider operation within the throttle region $40-80 \%$, which corresponds to surge speeds of $1.6-4.8 \mathrm{~m} / \mathrm{s}$. Then, by declaring that $30 \%$ throttle corresponds to zero surge speed, and by using the steady-state output (surge speed) data vector

$$
\mathbf{u}=[0,1.6,2.3,3.2,3.9,4.8]^{\top}
$$

with the corresponding scaled input (throttle) data vector

$$
\boldsymbol{\sigma}=[0,0.2,0.4,0.6,0.8,1]^{\top},
$$

the following analytical relationship was obtained through least-squares curve fitting against a third order polynomial

$$
\sigma(u)=-0.0078 u^{3}+0.0720 u^{2}+0.0428 u-0.0017,
$$

which is valid for zero rudder and positive surge speeds. Figure 8 illustrates the maneuver map encapsulated by (16), and shows its correspondence with the data from (14) and (15). As can be seen, this input-output relationship comes close to being linear for high speeds, which is due to the fact that the nonlinear effects of the throttle input mainly competes with the nonlinear effects of the hydrodynamic damping at such speeds.

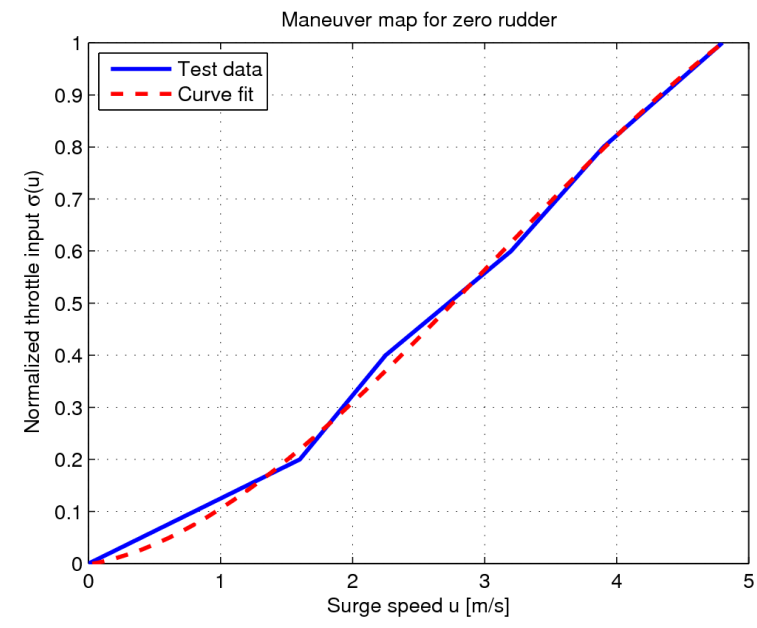

Figure 8: The maneuver map obtained through steadystate USV experiments.

Also, note that the relationship between $\sigma(u) \in[0,1]$ and the actual throttle $\tau(\sigma(u))$ is equal to

$$
\tau(\sigma(u))=100(0.5 \sigma(u)+0.3)
$$

since $\sigma(u)=0$ corresponds to $30 \%$ throttle and $\sigma(u)=$ 1 corresponds to $80 \%$ throttle. Thus, (16) and (17) tells us that if a surge speed of $3.2 \mathrm{~m} / \mathrm{s}$ is desired, a throttle of $60 \%$ must be applied. For control design purposes, any desired surge speed value that is within the speed range of the maneuver map nominally constitutes a feasible value.

Agility Test One way to determine the maximum agility of a vehicle is to record the response of the maneuver states to steps in the control inputs from $0 \%$ to $100 \%$. Such step response analysis determines how fast the vehicle is able to move in the maneuver space. For our vehicle, the agility test was performed as a step in the throttle for zero rudder, which resulted in a surge speed response as shown in the top part of Figure 9. The figure shows two distinct regions of behavior, i.e., one region where the speed climbs fast to $5 \mathrm{~m} / \mathrm{s}$ (displacement region) and another where it increases more slowly up toward $10 \mathrm{~m} / \mathrm{s}$ (semi-displacement to planing regions). As already mentioned, it was decided to just consider speeds below $5 \mathrm{~m} / \mathrm{s}$ (10 knots) since it is very difficult to precision control the vehicle speed outside the displacement region without installing additional control surfaces. Hence, the maximum USV speed $U_{\max }$ was set to $5 \mathrm{~m} / \mathrm{s}$.

The bottom part of Figure 9 shows the surge speed response in the displacement region together with an approximation. This approximation is not achieved by 

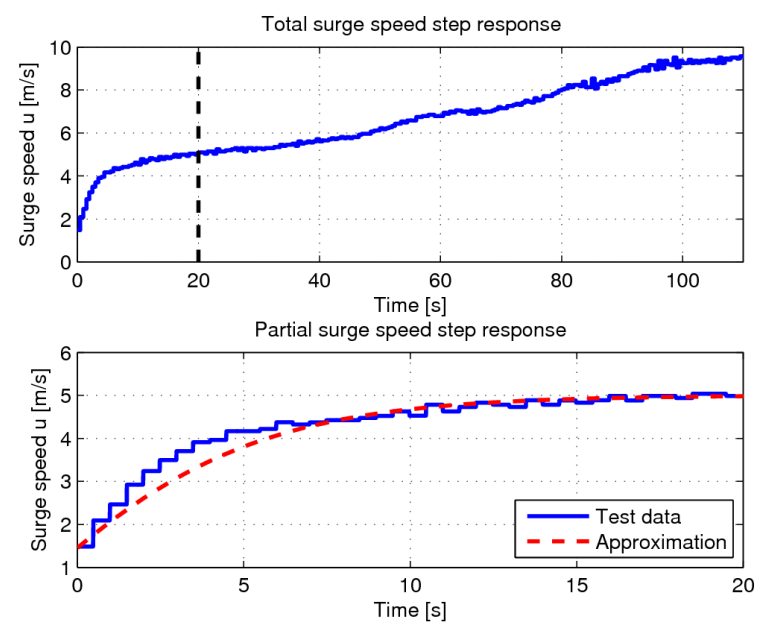

Figure 9: Top: Complete surge speed step response. Bottom: Actual and approximated displacement region responses.

using a low-pass filter with a time constant, as is common when approximating step responses, but rather as a sigmoid-like tanh function with a dynamic input state, i.e., as

$$
u(t)=\triangle_{\mathrm{u}} \tanh \left(\frac{\rho(t)}{\triangle_{\mathrm{u}}}\right)
$$

where $\dot{\rho}(t)=\alpha(t)$ with $\rho(0)=\triangle_{\mathrm{u}} \tanh ^{-1}\left(\frac{u(0)}{\triangle_{\mathrm{u}}}\right)$, and $\triangle_{\mathrm{u}}>0$ is a scaling variable that renders the tanh function magnitude-invariant, as opposed to a low-pass filter implementation, which is not magnitude-invariant. The variable $\alpha(t)$ thus represents an agility parameter indicating how fast transitions can be made between maneuver states, and the specific value of $\alpha(t)$ obtained through a step-response test involving zero to maximum control input then represents the maximum attainable agility $\alpha_{\max }$, i.e., $\alpha(t) \in\left\langle 0, \alpha_{\max }\right]$. This maximum value embeds information about all the dynamic phenomena occurring between the actuator control input and the navigation system output (e.g., motor dynamics, actuator dynamics, vessel-ocean dynamics, sensor dynamics, etc.) without the need for detailed modeling of these intermediate dynamic systems. Specifically, the maximum agility parameter corresponding to Figure 9 was found to be $\alpha_{\max }=0.7$, while $\triangle_{\mathrm{u}}=5$ equals the considered speed range. For control design purposes, any speed reference signal corresponding to an $\alpha(t)$ below $\alpha_{\max }$ nominally constitutes a feasible signal rate-wise.

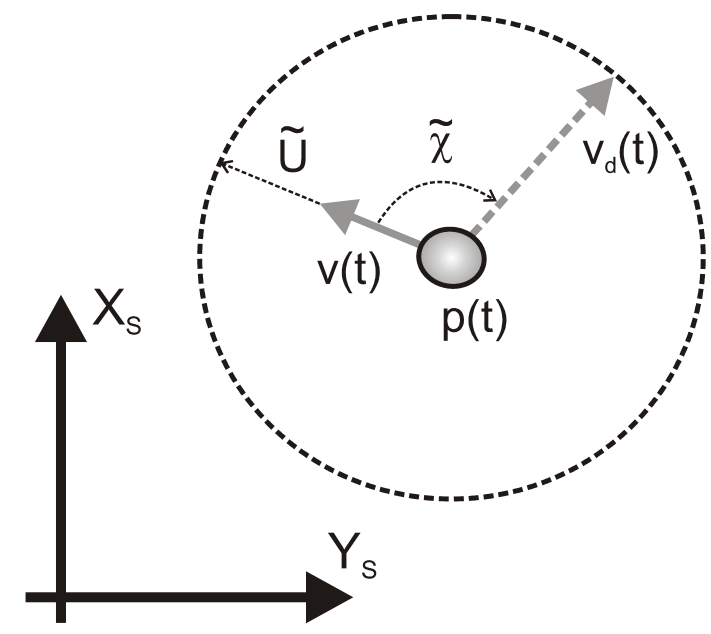

Figure 10: A polar coordinate decomposition of the velocity error $\tilde{\mathbf{v}}(t)$ into a speed error $\tilde{U}$ and a course error $\tilde{\chi}$.

\subsubsection{Surge Speed Controller}

Since we are dealing with an underactuated USV, the sway speed $v(t)$ cannot be directly controlled. Consequently, the desired velocity commanded by the guidance system must be divided between surge speed and yaw rate controllers in a polar coordinate fashion. Such a scheme means that the surge speed controller becomes responsible for controlling the size of the USV velocity $\mathbf{v}(t)$ while the yaw rate controller is responsible for controlling the direction of the velocity, see Figure 10. Note that the desired velocity $\mathbf{v}_{\mathrm{d}}(t)$ in this figure corresponds to the assigned velocity $\mathbf{v}(t)$ in Figure 4 .

Denoting the speed (velocity size) error as

$$
\tilde{U}(t) \triangleq U_{\mathrm{d}}(t)-U(t),
$$

where $U_{\mathrm{d}}(t) \triangleq\left|\mathbf{v}_{\mathrm{d}}(t)\right|$ with $\mathbf{v}_{\mathrm{d}}(t)$ as in (6), the objective of the speed control becomes

$$
\lim _{t \rightarrow \infty} \tilde{U}(t)=0
$$

which we need to rewrite in terms of a corresponding control objective for the surge speed. Since $U(t)=$ $|\mathbf{v}(t)|=\sqrt{u(t)^{2}+v(t)^{2}}$ and (20) states that our goal is to have $U(t) \rightarrow U_{\mathrm{d}}(t)$, we get that $\sqrt{u(t)^{2}+v(t)^{2}} \rightarrow$ $U_{\mathrm{d}}(t)$ or equivalently that $u(t) \rightarrow \sqrt{U_{\mathrm{d}}(t)^{2}-v(t)^{2}}$. Hence, we define a desired surge speed as

$$
u_{\mathrm{d}}(t) \triangleq \sqrt{U_{\mathrm{d}}(t)^{2}-v(t)^{2}},
$$

which is valid when assuming $U_{\mathrm{d}}(t) \geq|v(t)|$ at all times. This assumption is highly realistic since in practice $|v(t)|$ is just a small fraction of $U(t)$ for straight-line 
motion at high speeds. Then denoting the surge speed error as

$$
\tilde{u}(t) \triangleq u_{\mathrm{d}}(t)-u(t)
$$

with $u_{\mathrm{d}}(t)$ as in (21), the objective of the surge speed controller becomes

$$
\lim _{t \rightarrow \infty} \tilde{u}(t)=0
$$

which together with an appropriate control objective for the yaw rate controller will enable the fulfillment of the target-tracking control objective (1).

However, the surge speed controller should not use $u_{\mathrm{d}}(t)$ directly as a reference signal. To ensure both static and dynamic feasibility in the computation of such a reference, information obtained from the maneuverability and agility tests can be employed in the following way:

1. The reference must always be constrained within the range of the maneuver map since it is not physically possible to track a speed that is larger than $U_{\max }$, which is the speed that corresponds to a maximum throttle input.

2. The reference must not change faster than what conforms to the maximum agility parameter $\alpha_{\max }$.

Consequently, when supplied a desired speed $U_{\mathrm{d}}(t) \leq$ $U_{\text {max }}$, corresponding to $u_{\mathrm{d}}(t) \leq u_{\max }$ when adjusting for the sway speed, a feasible reference both magnitude- and rate-wise can be computed by

$$
u_{\mathrm{r}}(t)=u_{\max } \tanh \left(\frac{\rho_{\mathrm{r}}(t)}{u_{\max }}\right)
$$

where $u_{\text {max }}$ represents the maximum attainable surge speed, and the dynamics of $\rho_{\mathrm{r}}(t)$ is given by

$$
\dot{\rho}_{\mathrm{r}}(t)=\alpha(t) \tanh \left(\frac{k_{\mathrm{p}, \tilde{\rho}} \tilde{\rho}(t)}{\alpha(t)}\right),
$$

where $\alpha(t) \in\left\langle 0, \alpha_{\max }\right], k_{\mathrm{p}, \tilde{\rho}}>0, \tilde{\rho}(t) \triangleq \rho_{\mathrm{d}}(t)-\rho_{\mathrm{r}}(t)$ where

$$
\rho_{\mathrm{d}}(t)=u_{\max } \tanh ^{-1}\left(\frac{u_{\mathrm{d}}(t)}{u_{\max }}\right)
$$

corresponds to the desired surge speed $u_{\mathrm{d}}(t)$, and with

$$
\rho_{\mathrm{r}}(0)=u_{\max } \tanh ^{-1}\left(\frac{u(0)}{u_{\max }}\right)
$$

accounting for the initial surge speed $u(0)$. Thus, $u_{\mathrm{r}}(t)$ functions as a feasibility filter between $u(t)$ and $u_{\mathrm{d}}(t)$, starting in $u(0)$ and tracking $u_{\mathrm{d}}(t)$ constrained by $\alpha(t) \leq \alpha_{\max }$. This filter is structurally identical with (18) and ensures feasible operation at all times by relying on recorded maneuverability and agility data embedded in $u_{\max }$ and $\alpha_{\max }$. Specifically, while (24) ensures maneuverability compliance, (25) ensures agility compliance. It is then the responsibility of the velocity control system to make $u(t)$ track $u_{\mathrm{r}}(t)$ such that (23) is fulfilled for a feasible $u_{\mathrm{d}}(t)$. However, if $u_{\mathrm{d}}(t)$ is infeasible somehow (either statically, dynamically, or both), it cannot be tracked in any case.

Having obtained the maneuver map constituted by Figure 8, a feasible reference speed $u_{\mathrm{r}}(t)$ can ideally be gained simply by commanding the throttle input corresponding to $\sigma\left(u_{\mathrm{r}}(t)\right)$, which is a pure feedforward control assignment. Naturally, such an assignment can only result in satisfactory performance for conditions similar to those for which the maneuver tests were performed. Consequently, feedback must also be added as part of the control strategy in order to achieve robustness against curve-fitting errors, off-nominal conditions, and disturbances. Hence, consider the following surge speed controller

$$
c\left(u_{\mathrm{r}}(t), \bar{u}(t)\right)=\sigma\left(u_{\mathrm{r}}(t)\right)+k_{\mathrm{p}, \overline{\mathrm{u}}} \bar{u}(t)+k_{\mathrm{i}, \overline{\mathrm{u}}} \int_{0}^{t} \bar{u}(\tau) \mathrm{d} \tau,
$$

where

$$
\bar{u}(t) \triangleq u_{\mathrm{r}}(t)-u(t)
$$

with $u_{\mathrm{r}}(t)$ as in $(24)$ and $k_{\mathrm{p}, \overline{\mathrm{u}}}>k_{\mathrm{i}, \overline{\mathrm{u}}}>0$. This controller thus consists of a feedforward term based on the maneuver map (16) and a PI feedback control term for robust and tight surge speed control. The corresponding throttle command becomes

$$
\tau_{\mathrm{c}}\left(u_{\mathrm{r}}(t), \bar{u}(t)\right)=100\left(0.5 c\left(u_{\mathrm{r}}(t), \bar{u}(t)\right)+0.3\right),
$$

which help ensure that

$$
\lim _{t \rightarrow \infty} \bar{u}(t)=0
$$

and thus that the surge speed control objective (23) can be feasibly fulfilled.

\subsubsection{Yaw Rate Controller}

As previously mentioned, the role of the yaw rate controller is to make the direction of the USV velocity match the direction of the desired velocity commanded by the guidance system. Thus, denoting the course (velocity direction) error as

$$
\tilde{\chi}(t) \triangleq \chi_{\mathrm{d}}(t)-\chi(t)
$$

where $\chi_{\mathrm{d}}(t) \triangleq \operatorname{atan} 2\left(\dot{y}_{\mathrm{d}}(t), \dot{x}_{\mathrm{d}}(t)\right)$ represents the desired course angle associated with $\mathbf{v}_{\mathrm{d}}(t)$ and $\chi(t)=$ $\operatorname{atan} 2(\dot{y}(t), \dot{x}(t))$ represents the actual USV course angle, the objective of the course control becomes

$$
\lim _{t \rightarrow \infty} \tilde{\chi}(t)=0
$$

which together with the control objective for the surge speed controller (23) enables the fulfillment of the target-tracking control objective (1). 
However, we do not calculate $\tilde{\chi}(t)$ according to (32) by using the explicit course angles. To avoid possible wraparound problems associated with such a method, $\tilde{\chi}(t)$ can be calculated directly by employing cross- and inner-product information about the velocities $\mathbf{v}_{\mathrm{d}}(t)$ and $\mathbf{v}(t)$. Specifically, we can extract $\sin (\tilde{\chi}(t))$ information from the cross product $\mathbf{v}(t) \times \mathbf{v}_{\mathrm{d}}(t)$ and $\cos (\tilde{\chi}(t))$ information from the inner product $\mathbf{v}(t)^{\top} \mathbf{v}_{\mathrm{d}}(t)$ for use in the direct calculation

$$
\tilde{\chi}(t)=\operatorname{atan} 2(\sin (\tilde{\chi}(t)), \cos (\tilde{\chi}(t))),
$$

see Figure 10. This method of deriving $\tilde{\chi}(t)$ is unorthodox and does not seem to have been reported in the marine literature before.

Since the considered target-tracking scenario only involves straight-line motion, the yaw rate controller does not require any feedforward terms, and thus no corresponding maneuverability and agility tests need to be performed. Consequently, the commanded rudder angle input can simply be chosen as the pure PI feedback controller

$$
\delta_{\mathrm{c}}(\tilde{r}(t))=k_{\mathrm{p}, \tilde{\mathrm{r}}} \tilde{r}(t)+k_{\mathrm{i}, \tilde{\mathrm{r}}} \int_{0}^{t} \tilde{r}(\tau) \mathrm{d} \tau
$$

with $k_{\mathrm{p}, \tilde{\mathrm{r}}}>k_{\mathrm{i}, \tilde{\mathrm{r}}}>0$ and

$$
\tilde{r}(t) \triangleq r_{\mathrm{d}}(t)-r(t)
$$

where

$$
r_{\mathrm{d}}(t)=r_{\mathrm{a}, \max } \tanh \left(\frac{k_{\mathrm{p}, \tilde{\chi}} \tilde{\chi}(t)}{r_{\mathrm{a}, \max }}\right)
$$

is employed as the desired yaw rate, with $r_{\mathrm{a} \text {, max }}$ representing the maximum yaw rate at which $\chi(t)$ is allowed to approach $\chi_{\mathrm{d}}(t)$, and $k_{\mathrm{p}, \tilde{\chi}}>0$ shaping this approach. Hence, $(37)$ ensures that $\chi(t)$ will rendezvous with $\chi_{\mathrm{d}}(t)$ in a controlled manner, while the smoothness of the approach depends on $k_{\mathrm{p}, \tilde{\chi}}$. Figure 11 shows how the desired yaw rate varies as a function of this gain, i.e., a large value results in a steep approach and vice versa.

Far from a traditional autopilot, the suggested yaw rate controller employs no explicit information about the USV heading angle $\psi(t)$, and controls $\tilde{\chi}(t)$ in a cascaded manner through inner loop control of $\tilde{r}(t)$.

\subsection{Total Motion Control System}

Summarizing the guidance and velocity control system development, we arrive at Figure 12. This figure illustrates the total motion control system resulting from the proposed designs of the previous sections. As can be seen, this paper contributes at the strategic and tactical levels of control, ultimately issuing throttle and rudder commands for the execution-level proportional

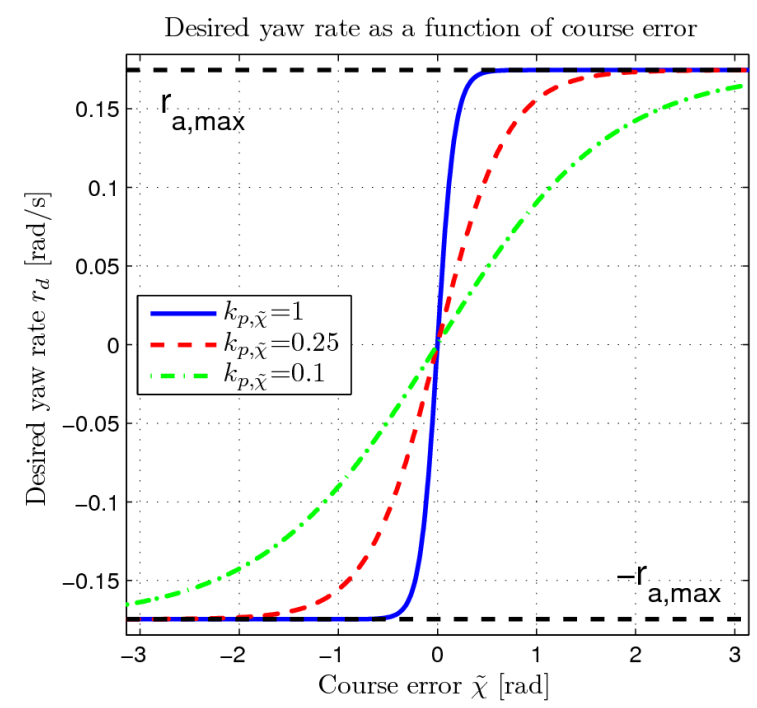

Figure 11: Profile of desired yaw rate as a function of course error for varying $k_{\mathrm{p}, \tilde{\chi}}$.

controllers governing the USV outboard engine. The developed motion control system enables underactuated USVs to track high-speed targets, especially those moving in a straight line. Its potential is illustrated through full-scale experiments in the next section.

\section{Full-Scale Experimental Results}

On Friday 1 August 2008, full-scale experiments were carried out in the Trondheimsfjord where the Kaasbøll USV was supposed to track the position of a virtual target travelling in a straight line at high speed. The environmental conditions during these experiments were far from the ideal conditions that were present on the day when the maneuverability and agility tests were carried out. Specifically, the ocean was visually estimated to be in sea state 3 (Faltinsen, 1990), which is pretty rough for a small vessel such as the Kaasbøll USV. Also, the wind was blowing at around 3.5 $\mathrm{m} / \mathrm{s}$ with gusts up to $6.5 \mathrm{~m} / \mathrm{s}$ during the experiments. These conditions were certainly right to test the developed motion control system and explore its performance and robustness.

In the particular experiment detailed here, the virtual target started about $90 \mathrm{~m}$ to the northeast of the USV, moving due north in a straight line at a speed of $U_{\mathrm{t}}=3 \mathrm{~m} / \mathrm{s}$. The USV started at rest with an initial heading of $145 \mathrm{deg}$. It was allowed a maximum approach speed of $U_{\mathrm{a} \text {, } \max }=1 \mathrm{~m} / \mathrm{s}$ with which to intercept the target, i.e., allowed to move with a maximum total speed of $U_{\mathrm{t}}+U_{\mathrm{a}, \max }=4 \mathrm{~m} / \mathrm{s}<U_{\max }$. Also, the USV was allowed a maximum approach yaw rate 


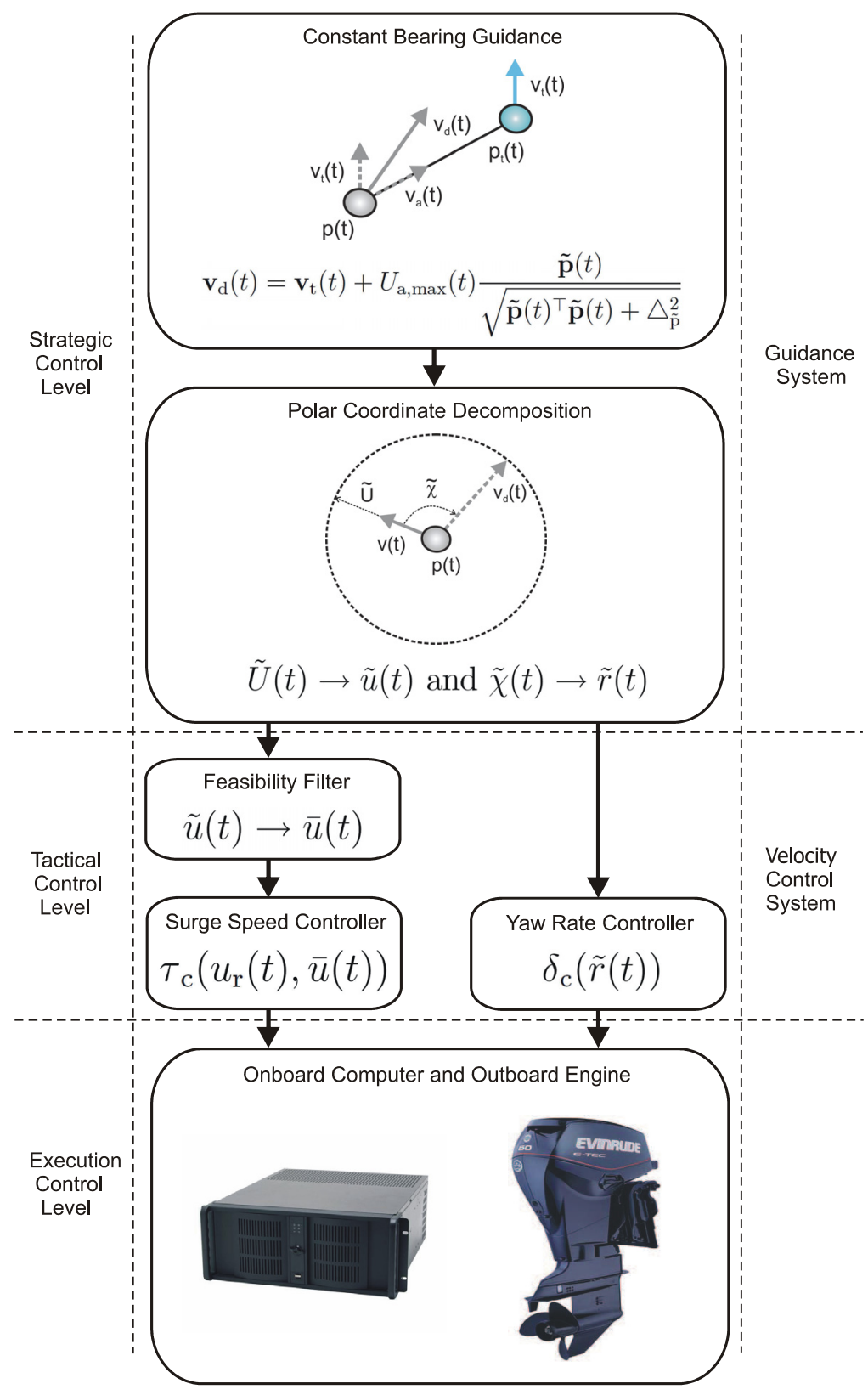

Figure 12: An illustration of the proposed motion control system capable of achieving high-speed target tracking for underactuated USVs. By replacing the guidance system components, other motion control scenarios can also be handled. 


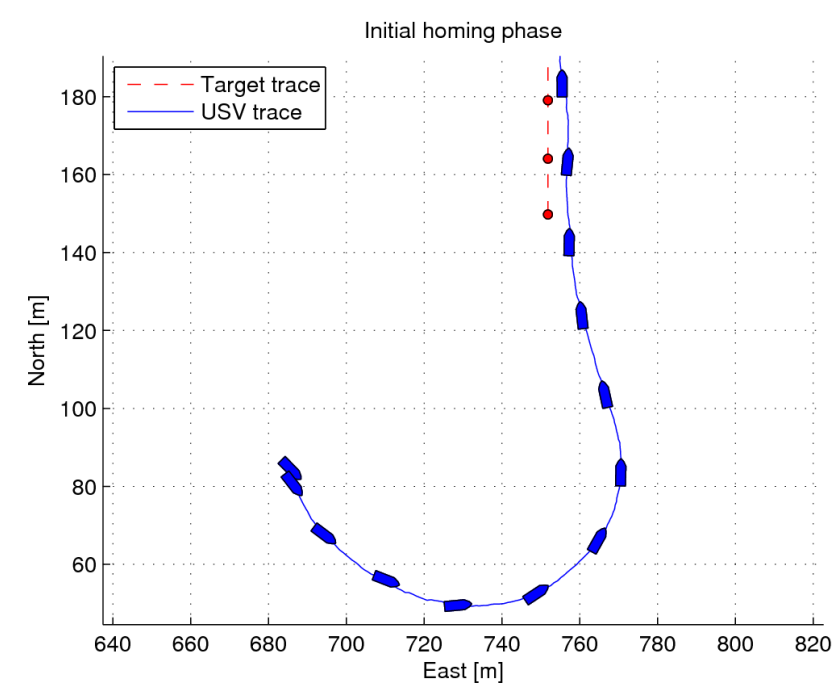

Figure 13: The USV is maneuvering onto an intercept course with the target.

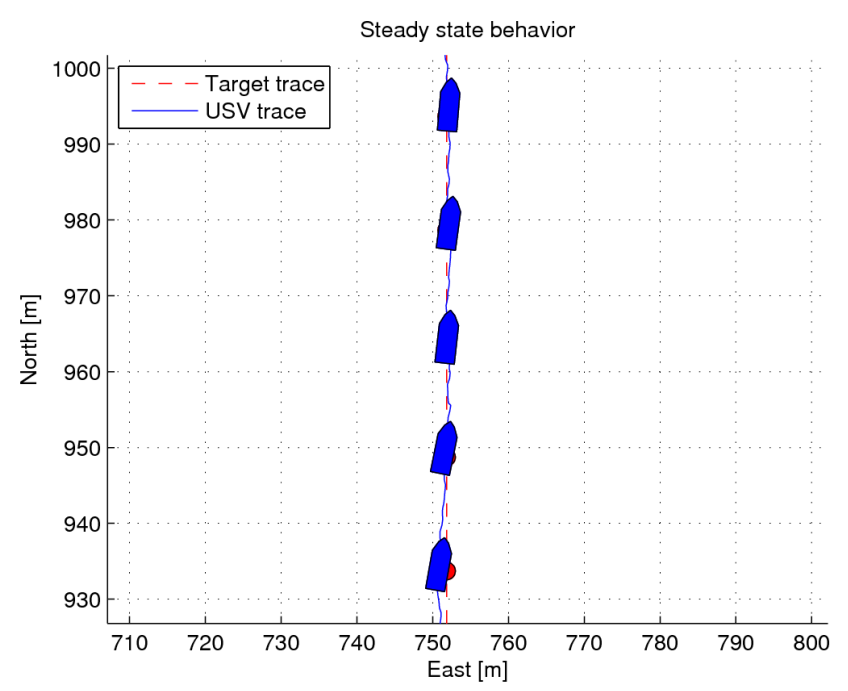

Figure 14: The USV has intercepted the target.

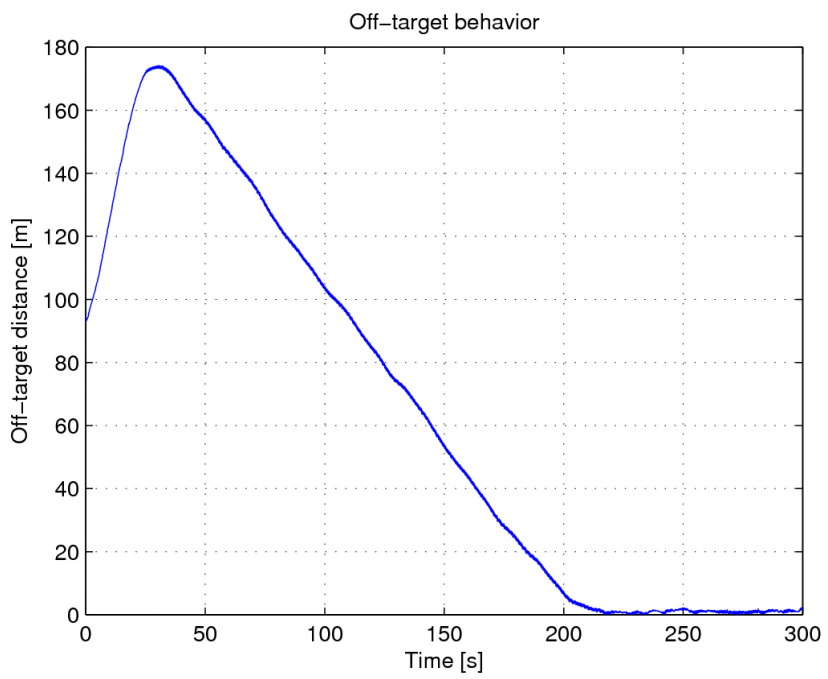

Figure 15: The distance to the target initially increases until the USV begins to move in the target direction and then finally converges smoothly to zero.
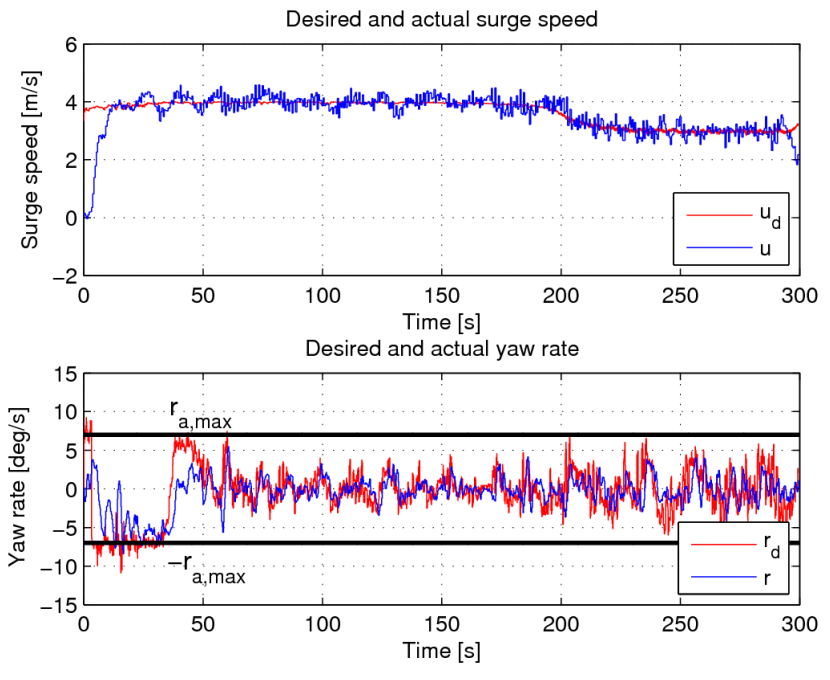

Figure 16: Top: The surge speed response of the Kaasbøll USV. Bottom: The yaw rate is seen to remain within the bounds of $r_{\mathrm{a}, \max }$. 
of $r_{\mathrm{a}, \max }=0.122 \mathrm{rad} / \mathrm{s}$ (equivalent to $7 \mathrm{deg} / \mathrm{s}$ ) with which to align its velocity with the desired velocity. Furthermore, the guidance system employed $\triangle_{\tilde{\mathrm{p}}}=10$ $m$, the surge speed reference filter used $\alpha=0.4<\alpha_{\max }$ and $k_{\mathrm{p}, \tilde{\rho}}=10$, the surge speed controller gains were chosen as $k_{\mathrm{p}, \tilde{\mathrm{u}}}=0.5$ and $k_{\mathrm{i}, \tilde{\mathrm{u}}}=0.05$, while the yaw rate controller employed $k_{\mathrm{p}, \tilde{\chi}}=0.5$ and the same PI gains as the surge speed controller.

Figure 13 shows the initial response of the USV as it powers up from rest and starts homing in on the target. The intercept approach appears natural and smooth. Figure 14 shows the steady-state performance of the USV after it has intercepted the target. The time evolution of the off-target distance $|\tilde{\mathbf{p}}(t)|$ is shown in Figure 15. As can be seen, the distance increases in the beginning while the USV is turning to achieve its intercept course. After about 30 seconds, the USV has finished turning and the distance to the target decreases with $1 \mathrm{~m} / \mathrm{s}$ until intercept takes place after approximately 220 seconds. The top part of Figure 16 shows that the surge speed quickly achieves $4 \mathrm{~m} / \mathrm{s}$ and then starts to track the reference speed with about 0.5 $\mathrm{m} / \mathrm{s}$ accuracy, which is acceptable given the sea state of the experiment. Also, the bottom part of Figure 16 shows that the yaw rate is kept within the limitation of $7 \mathrm{deg} / \mathrm{s}$ and tracks the reference well given the environmental conditions. Furthermore, Figure 17 shows that the commanded throttle and rudder are well within their bounds, while Figure 18 shows how the targettracking response becomes less tight with a smaller $r_{\mathrm{a}, \max }=0.087 \mathrm{rad} / \mathrm{s}$ (equivalent to $5 \mathrm{deg} / \mathrm{s}$ ). The green lines of this figure represent the line-of-sight vector between the USV and its target, illustrating how the application of constant bearing guidance leads to stabilization of the LOS angle, and also why the approach sometimes is referred to as parallel navigation. In sum, these results show that the USV motion control system performs very well despite tough conditions.

\section{Conclusions and Further Work}

This paper has addressed the subject of straight-line target tracking for unmanned surface vehicles (USVs). Specifically, the work presented the design of a motion control system that enables an underactuated USV to track a target which moves in a straight line at high speed. The motion control system includes a guidance law originally developed for interceptor missiles, as well as a new type of velocity control which is inspired by maneuverability and agility concepts found in literature on fighter aircraft. In fact, several novel concepts were introduced in the design, and its performance was successfully illustrated through full-scale target-tracking experiments in the Trondheimsfjord.
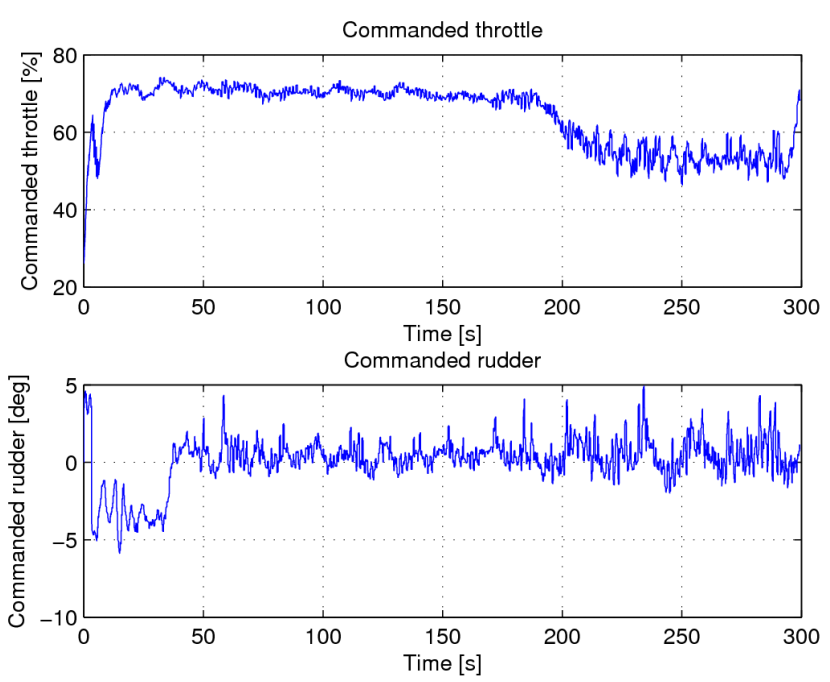

Figure 17: The commanded actuator inputs remain well within their bounds.

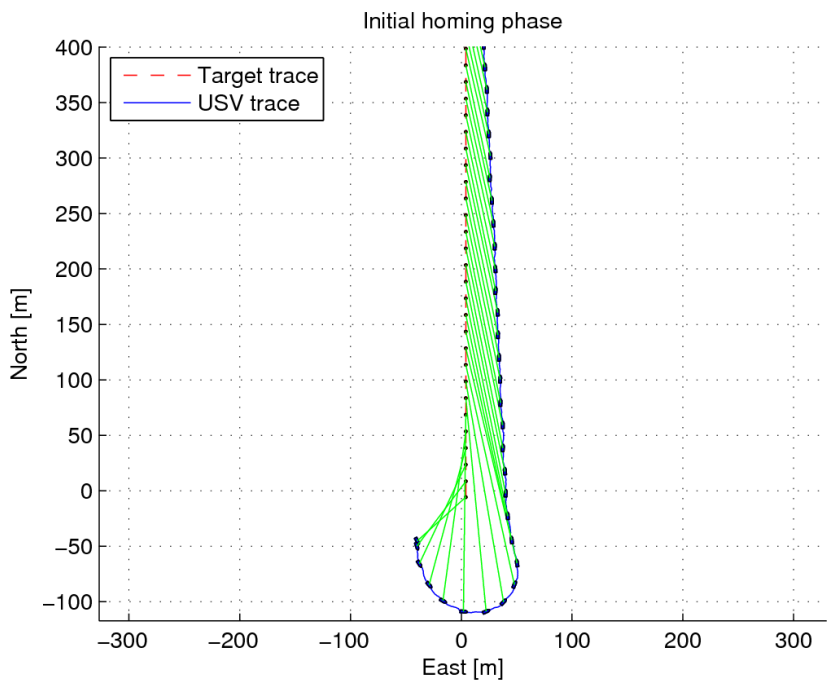

Figure 18: An alternate intercept run with less tight motion control. 


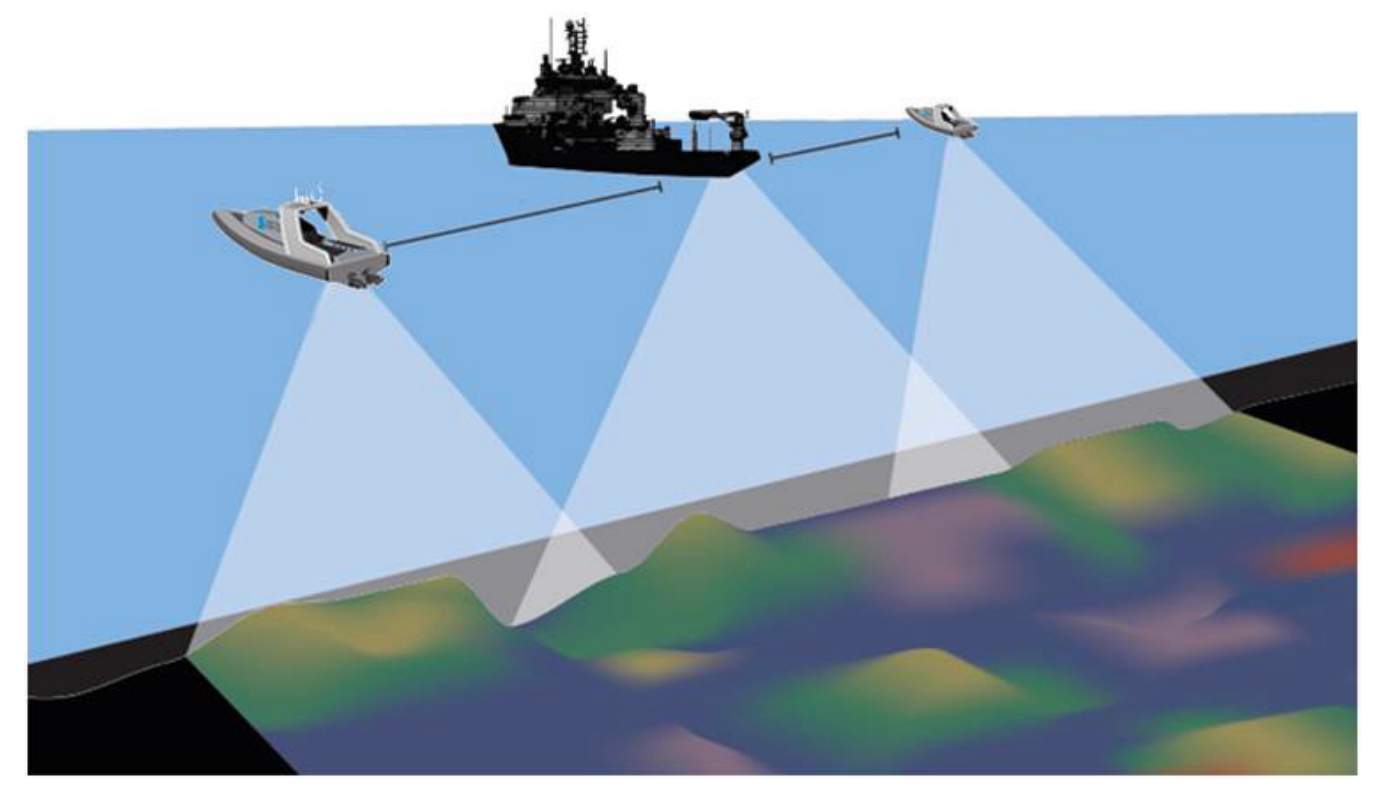

Figure 19: USV-assisted seabed mapping entails faster and cheaper operations since the capacity of the main survey vessel is augmented by that of a fleet of USVs. Courtesy of Maritime Robotics.

This work represents a first step toward the development of new motion control systems that take advantage of the maneuvering abilities of small and highpowered USVs.

Further work includes extending the current motion control system to also handle circular target motion, which involves performing additional maneuverability and agility tests to find the maneuver map between the rudder input and the yaw rate output for feedforward use in the yaw rate controller. Such an enhanced motion control system can for instance be used to achieve formation control with a group of underactuated USVs tracking the motion of a manned leader vessel which has a specific geometric formation pattern associated with it, see Figure 19.

\section{Acknowledgments}

This work was supported by the Norwegian Research Council through the Centre for Ships and Ocean Structures and through the MAROFF grant 175977: Unmanned Surface Vehicle. The authors would like to thank the reviewers for their constructive comments, while a special thanks goes to Arild Hepsø, Eirik E. Hovstein, and Stein Johansen at Maritime Robotics for their help with the full-scale experiments. This paper is a tribute to the Kaasbøll USV that was used in the experiments, but which has since been dismantled and replaced by other test platforms.

\section{References}

Aguiar, A. P. and Hespanha, J. P. Trajectorytracking and path-following of underactuated autonomous vehicles with parametric modeling uncertainty. IEEE Transactions on Automatic Control, 2007. 52(8):1362-1379.

Beck, J. A. and Cord, T. J. A framework for analysis of aircraft maneuverability. In Proceedings of the AIAA Atmospheric Flight Mechanics Conference, Baltimore, Maryland, USA. 1995.

Benjamin, M. R., Leonard, J. J., Curcio, J. A., and Newman, P. M. A method for protocol-based collision avoidance between autonomous marine surface craft. Journal of Field Robotics, 2006. 23(5):333346.

Bertram, V. Unmanned surface vehicles - A survey. In Skibsteknisk Selskab, Copenhagen, Denmark. 2008 .

Breivik, M. and Fossen, T. I. Path following for marine surface vessels. In Proceedings of the OTO'04, Kobe, Japan. 2004 .

Breivik, M. and Fossen, T. I. Applying missile guidance concepts to motion control of marine craft. In Proceedings of the 7th IFAC CAMS, Bol, Croatia. 2007 .

Breivik, M. and Fossen, T. I. Guidance laws for planar motion control. In Proceedings of the $C D C^{\prime} 08$, Cancun, Mexico. 2008 . 
Breivik, M., Strand, J. P., and Fossen, T. I. Guided dynamic positioning for fully actuated marine surface vessels. In Proceedings of the 6th IFAC MCMC, Lisbon, Portugal. 2006 .

Brown, N. Not just a remote possibility: USVs enter the fray. Jane's Navy International, 2004. 109(1):1419 .

Børhaug, E. and Pettersen, K. Y. Cross-track control for underactuated autonomous vehicles. In Proceedings of the CDC-ECC'05, Seville, Spain. 2005.

Caccia, M. Autonomous surface craft: Prototypes and basic research issues. In Proceedings of the MED'06, Ancona, Italy. 2006 .

Caccia, M., Bibuli, M., Bono, R., and Bruzzone, G. Basic navigation, guidance and control of an unmanned surface vehicle. Autonomous Robots, 2008a. 25(4):349-365.

Caccia, M., Bruzzone, G., and Bono, R. A practical approach to modeling and identification of small autonomous surface craft. IEEE Journal of Oceanic Engineering, 2008b. 33(2):133-145.

Cooper, S. L., Newborn, D. A., and Norton, M. R. New paradigms in boat design: An exploration into unmanned surface vehicles. In Proceedings of the $A U$ VSI Unmanned Systems, Lake Buena Vista, Florida, USA. 2002 .

Corfield, S. J. and Young, J. M. Unmanned surface vehicles - Game changing technology for naval operations. In G. N. Roberts and R. Sutton, editors, Advances in Unmanned Marine Vehicles, pages 311328. The Institution of Electrical Engineers, 2006.

DeGarmo, M. and Nelson, G. M. Prospective unmanned aerial vehicle operations in the future national airspace system. In Proceedings of the 4 th AIAA ATIO Forum, Chicago, Illinois, USA. 2006

Do, K. D. and Pan, J. Underactuated ships follow smooth paths with integral actions and without velocity measurements for feedback: Theory and experiments. IEEE Transactions on Control Systems Technology, 2006. 14(2):308-322.

Doucy, O. and Ghozlan, F. Advanced functions for USV. In Proceedings of the ATMA International Autonomous Surface Ship Symposium, Paris, France. 2008 .

Draper, C. S. Guidance is forever. Navigation, 1971. $18(1): 26-50$.
Ebken, J., Bruch, M., and Lum, J. Applying unmanned ground vehicle technologies to unmanned surface vehicles. In Proceedings of SPIE 5804: Unmanned Ground Vehicle Technology VII, Orlando, Florida, USA. 2005 .

Faltinsen, O. M. Sea Loads on Ships and Offshore Structures. Cambridge University Press, 1990.

Faltinsen, O. M. Hydrodynamics of High-Speed Marine Vehicles. Cambridge University Press, 2005.

Fossen, T. I. Marine Control Systems: Guidance, Navigation and Control of Ships, Rigs and Underwater Vehicles. Marine Cybernetics, 2002.

Fossen, T. I. A nonlinear unified state-space model for ship maneuvering and control in a seaway. Journal of Bifurcation and Chaos, 2005. 15(9):2717-2746.

Fredriksen, E. and Pettersen, K. Y. Global $\kappa$ exponential way-point maneuvering of ships: Theory and experiments. Automatica, 2006. 42(4):677-687.

Gibbons, T. D. and Wilson, P. A. Operating a remotely controlled yacht at very large distances. In Proceedings of the ATMA International Autonomous Surface Ship Symposium, Paris, France. 2008.

Hook, D. J. Development of unmanned surface vehicles. In Proceedings of the World Maritime Technology Conference, London, UK. 2006 .

Kongsberg Maritime. Kongsberg K-Pos DP dynamic positioning system. 2006. Report no. 301093/B.

Kongsberg Seatex. Datasheet Seapath 20 NAV. 2006.

Larson, J., Bruch, M., Halterman, R., Rogers, J., and Webster, R. Advances in autonomous obstacle avoidance for unmanned surface vehicles. In Proceedings of the AUVSI Unmanned Systems North America, Washington D.C., USA. 2007 .

LaValle, S. M. Planning Algorithms. Cambridge University Press, 2006.

Loe, Ø. A. G. Collision Avoidance for Unmanned Surface Vehicles. Master's thesis, Norwegian University of Science and Technology, 2008.

Majohr, J. and Buch, T. Modelling, simulation and control of an autonomous surface marine vehicle for surveying applications Measuring Dolphin MESSIN. In G. N. Roberts and R. Sutton, editors, Advances in Unmanned Marine Vehicles, pages 329-351. The Institution of Electrical Engineers, 2006. 
Naeem, W., Xu, T., Sutton, R., and Tiano, A. The design of a navigation, guidance, and control system for an unmanned surface vehicle for environmental monitoring. Proceedings of the Institution of $\mathrm{Me}$ chanical Engineers, Part M: Journal of Engineering for the Maritime Environment, 2008. 222(2):67-79.

Navy, U. S. The Navy unmanned surface vehicle (USV) master plan. 2007.

Paranjape, A. A. and Ananthkrishnan, N. Combat aircraft agility metrics - A review. Journal of Aerospace Sciences and Technologies, 2006. 58(2):1-12.

Perez, T., Sørensen, A. J., and Blanke, M. Marine vessel models in changing operational conditions - A tutorial. In Proceedings of the 14th IFAC SYSID, Newcastle, Australia. 2006 .

Portmann, H. H., Cooper, S. L., Norton, M. R., and Newborn, D. A. Unmanned surface vehicles: Past, present, and future. Unmanned Systems, 2002. $20(5): 32-37$.

Sciavicco, L. and Siciliano, B. Modelling and Control of Robot Manipulators. Springer-Verlag London Ltd., 2002.

Shneydor, N. A. Missile Guidance and Pursuit: Kinematics, Dynamics and Control. Horwood Publishing Ltd., 1998.

Skjetne, R., Fossen, T. I., and Kokotović, P. V. Robust output maneuvering for a class of nonlinear systems. Automatica, 2004a. 40(3):373-383.

Skjetne, R., Smogeli, Ø. N., and Fossen, T. I. A nonlinear ship manoeuvering model: Identification and adaptive control with experiments for a model ship. Modeling, Identification and Control, 2004b. 25(1):3-27.

Sørensen, A. J., Leira, B., Strand, J. P., and Larsen, C. M. Optimal setpoint chasing in dynamic positioning of deep-water drilling and intervention vessels. International Journal of Robust and Nonlinear Control, 2001. 11:1187-1205.

Valavanis, K. P., Gracanin, D., Matijasevic, M., Kolluru, R., and Demetriou, G. A. Control architectures for autonomous underwater vehicles. IEEE Control Systems Magazine, 1997. 17(6):48-64.

Withington, T. No crew onboard! Armada International, 2008. 32(3):18-26. 